\begin{tabular}{|c|l|}
\hline Title & High-Y ield Single Photon Source Using Gated Spontaneous Parametric Downconversion \\
\hline Author(s) & Takeuchi, Shigeki; OKamoto, Ryo; Sasaki, Keiji \\
\hline Citation & $\begin{array}{l}\text { A pplied Optics, 43(30), 5708-5711 } \\
\text { https://doi.org/40.1364/A0.43.005708 }\end{array}$ \\
\hline Issue Date & 2004 10-20 \\
\hline Doc URL & http://hdl.handle.net/2115/49844 \\
\hline Rights & ○ 2004 Optical Society of A merica \\
\hline Type & article \\
\hline File Information & A 043-30_5708-5711.pdf \\
\hline
\end{tabular}

Instructions for use 


\title{
High-yield single-photon source using gated spontaneous parametric downconversion
}

\author{
Shigeki Takeuchi, Ryo Okamoto, and Keiji Sasaki
}

\begin{abstract}
The construction of a single-photon source by use of gated parametric fluorescence is reported with the measurement results of the photon number distribution. A beamlike twin-photon method is used in order to achieve high-collection efficiency. The estimated probability $P(1)$ to find a single photon in a collimated output pulse is $26.5 \%$ at a repetition rate of $10 \mathrm{kHz}$ when the effective quantum efficiency of $27.4 \%$ in the detection setup is compensated. (C) 2004 Optical Society of America

OCIS codes: $270.0270,270.5290,270.6570,030.5260,230.6080$.
\end{abstract}

\section{Introduction}

One of the most important applications of the singlephoton source is quantum cryptography. ${ }^{1}$ Up until now, most quantum cryptography experiments have used weak coherent light as a photon source. ${ }^{2}$ In those experiments, the average photon number was kept as small as 0.1 in order to keep the probability of generating two photons in one pulse smaller than 0.005 . However, this meant that $90 \%$ of the pulses were vacuum states and could not be used to send any information. Therefore single-photon sources that can output single-photon states with a high probability while having only small percentages of twophoton states are very important for quantum cryptography. Such single-photon sources are also important for quantum information processing.

There has been extensive research to produce single-photon pulses by use of a single light emitter. Experimental demonstrations have been performed with single molecules, ${ }^{3,4}$ single color centers in diamond, 5 and single quantum $\operatorname{dots}^{6}$ optically pumped by short-pulse lasers. For electrically driven solidstate single-photon sources, the pioneering work us-

S. Takeuchi (takeuchi@es.hokudai.ac.jp) is with the Japanese Science and Technology Corporation, Precursory Research for Embryonic Science and Technology Project, Research Institute for Electronic Science, Hokkaido University, Kita 12 Nishi 6, Kita-ku, Sapporo, Hokkaido 060-0812, Japan. R. Okamoto and K. Sasaki are with the Research Institute for Electronic Science, Hokkaido University, Kita 12 Nishi 6, Kita-ku, Sapporo, Hokkaido 060-0812, Japan.

Received 24 November 2003; revised manuscript received 28 April 2004; accepted 25 June 2004.

0003-6935/04/305708-04\$15.00/0

(C) 2004 Optical Society of America ing turnstile devices at $50 \mathrm{mK}^{7}$ was recently followed by a demonstration of operation at $4.2 \mathrm{~K} .^{8}$ In those methods, however, photons are emitted in all directions, and it is difficult to collect the photons with high efficiency. This means that effectively the percentage of single-photon pulses will be small and that of vacuum states will be large. To the best of our knowledge, the highest probability $P(1)$ of a single photon at an output port where single photons are collimated was less than $10 \%$ in such devices. Recently, Pelton et al..$^{9}$ reported an excellent experimental result using a quantum dot embedded in a semiconductor micropost microcavity, where the efficiency of emitting a single-mode traveling wave inside the cavity was 38\%. However, the overall detection efficiency was only $3 \%$, owing to the technical difficulties of collecting the single photons and filtering them out from background photons.

The generation of single-photon states with spontaneous parametric downconversion (SPDC) is an alternative approach that was first mentioned by Hong and Mandel. ${ }^{10}$ It was theoretically followed by Yuen, ${ }^{11}$ who studied the parametric processes with measurement feedback for the generation of nearphoton-number eigenstates. The idea was experimentally followed by several groups..$^{12-14}$ However, these experiments focused on the observation of subPoissonian photon statistics and not the demonstration of a true single-photon source. Therefore they simply measured the correlation function $g^{(2)}(\tau)$ and determined the Fano factor $F=(\Delta n)^{2} /\langle n\rangle$. Both properties characterize the average noise level of the photon current, but the photon number distribution cannot be derived from only these parameters. ${ }^{15}$

In this paper, we present a single-photon source based on gated spontaneous parametric downconver- 


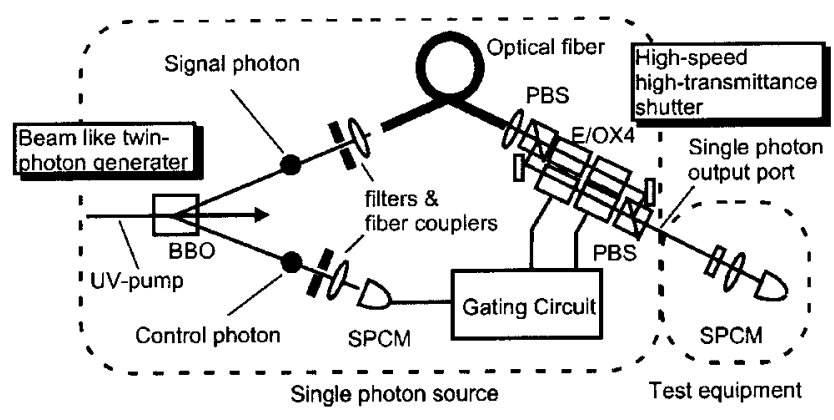

Fig. 1. Schematic of the experimental setup. A signal photon and a control photon are created at the same time in a $\beta$-barium borate crystal. The control photons were detected directly by a single-photon detector, while the signal photons were guided into an optical fiber delay line. Then the signal photons were gated by the fast optical shutter. This shutter opens only when the first detection event of the control photon is observed in each pulse (time window of $100 \mu \mathrm{s}$ ) and blocks other photons in the same pulse.

sion (G-SPDC). In contrast to earlier work, ${ }^{12}$ our G-SPDC source is run in a new regime in which a single photon is emitted in each time window with high probability and a well-defined repetition rate. Furthermore, the quality of our single-photon source is measured by characterizing the photon number distribution of its output.

\section{Experimental Setup and Data Analysis}

A schematic of our single-photon source is shown in Fig. 1. When a cw UV pump beam at $351.1 \mathrm{~nm}$ is incident to a nonlinear crystal fulfilling the phasematching condition, two pairs of single visible photons, control photons and signal photons, are created at the same time through the process of SPDC.16 We adjusted the experimental parameters to have a sufficient number of photon pairs created in a pulse with $100-\mu$ s pulse width. In our experiment, an average of eight control photons is detected within the pulse width. Following the first photon detection of a control photon in the pulse, the gate timing controller opens the shutter for a short time $(50 \mathrm{~ns})$. This means that only the first signal photon in the pulse can pass through the shutter and be emitted. After the emission, the shutter is closed to block the other photons in the pulse. The repetition of this procedure every $100 \mu \mathrm{s}$ results in a repetition rate of 10 $\mathrm{kHz}$ in our experiment. We have reported that parametric fluorescence can be emitted into two small spots and that the ratio of coincident counts to single counts can be larger than 0.8. ${ }^{17}$ In this experiment, we adopted this condition to increase the collection efficiency of photons and also focused the pump laser beam softly with a convex lens to obtain a high yield of parametric fluorescence. ${ }^{18}$

During the time it takes to control the shutter according to the detection signal of the control photon (which is $150 \mathrm{~ns}$ in our experiment), the signal photon should be stored with minimal loss. For this purpose, we adopted an optical delay line using a multimode optical fiber with a transmittance of $50 \%$, including the loss that occurs at the input/output fiber couplers and at the UV cut filters inserted in front of the fiber coupler.

We introduced a fast optical shutter with high transmission in this experiment. For fast optical switching, optical shutters using electro-optic modulators (EOMs) are generally used. However, such EOM modulators are polarization dependent and are not appropriate for the signal photon output from the multimode fiber-delay line in which the polarization has been randomized. Therefore we used the optical setup for the shutter shown in Fig. 1. To begin with, the first polarizing beam splitter transforms the signal photon into a superposition of the states in each of the paths according to its polarization. Then the EOMs (LM0202, Linos Photonics, Göttingen, Germany) in both paths rotate the polarization by $90^{\circ}$ only when the shutter is controlled to be closed. Finally, the two components are combined again by the second polarizing beam splitter. We used two EOMs for each path in order to minimize the shutter opening time and to avoid the restriction for repetition time given by the EOM drivers (LIV-8, Linos). In the experiment, the shutter opening time was set to $50 \mathrm{~ns}$ with a transmittance of $83 \%$. With this setup we achieved an overall effective transmittance of $41 \%$ for signal photons.

To find the photon number distribution of the output state of our single-photon source (in multimode), we measured the number of photons in each output pulse $(100 \mu \mathrm{s})$ with a photon number analyzer consisting of a single-photon counting module (SPCMAQ-FC, PerkinElmer, Wellesley, Massachusetts) and a photon counter (SR-400, Stanford Research Systems, Sunnyvale, California). The real photon number distribution of the output modified by the losses caused by the limited photon-detection efficiency of the photon number analyzer is as follows ${ }^{10}$ :

$$
P^{\prime}(i)=\sum_{j \geq i} \frac{j !}{(j-i) ! i !} \eta^{i}(1-\eta)^{j-i} P(j),
$$

where $\eta, P(j)$, and $P^{\prime}(i)$ are the effective quantum efficiency of the analyzer, the probability of having $j$ photons in the output, and the percentage of the events where the analyzer recorded $i$ signals during the pulse duration, respectively. We estimated the photon number distribution $P(j)$ of the output state by applying Eq. (1) to the experimental values of $P^{\prime}(i)$ for $i=0$ to 2 and the estimated effective quantum efficiency of $\eta=0.274 \pm 0.019$, taking into account the estimated quantum efficiency of $70 \pm 5 \%$ for SPCM and experimentally measured optical losses caused by the coupling lens and a mirror (transmittance, 90.2\%), the filter for the stray photons (transmittance, 49.2\%) and the fiber coupler (transmittance, 88.2\%). As a result, the photon-detection efficiency of the photon number analyzer is estimated to be $27.4 \%$. Note that we compensated the photon-detection efficiency of the photon number analyzer at the output, but we did not compensate the optical loss in the experimental setup of the G-SPDC single-photon source.

In our estimation of $P^{\prime}(i)$, the dark count of the 


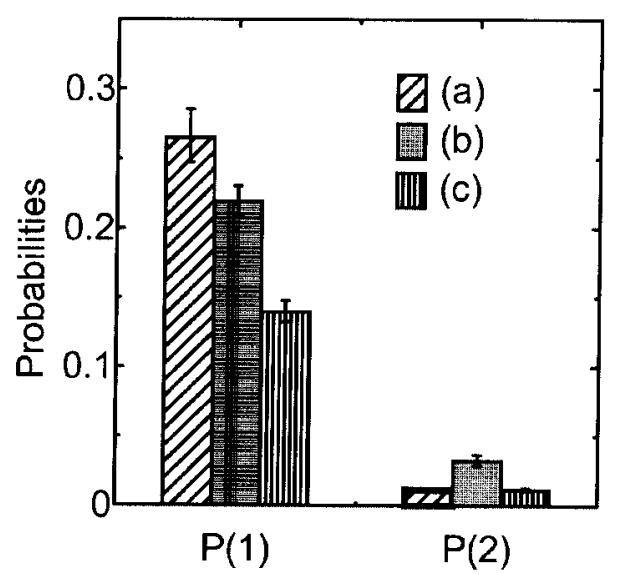

Fig. 2. (a) Photon number distribution of the output state. $P(1)$ and $P(2)$ show the probability of finding one or two photons in an output pulse $(100 \mu \mathrm{s})$, respectively. We counted the number of detection events in each pulse and estimated a photon number distribution at the output of our source, as described in the text. (b) Photon number distribution of weak coherent light with the same average photon number of (a). (c) Photon number distribution of weak coherent light with the same $P(2)$ of (a).

detector of 100 counts per second was also subtracted from the original data. The number of photons that are not counted during the dead time (50 ns) of the photon counter was also compensated under the assumption that the SPDC has Poisson distribution in multimode. ${ }^{19}$

\section{Results and Discussions}

The photon number distribution obtained in our experiment is shown by the bars (a) in Fig. 2. These $P(j)$ are estimated by use of Eq. (1) from the raw photon counting data $P^{\prime}(0)=0.9199, P^{\prime}(1)=0.0794$, and $P^{\prime}(2)=0.0005$ with the measurement of $10^{5}$ output pulses. The probability $P(1)$ to have a single photon in a pulse is $0.265 \pm 0.02$, which is more than three times larger than the previously reported value, to the best of our knowledge. The probability $P(2)$ to have two photons in a pulse was estimated to be $0.011 \pm 0.001$. The average photon number of the output in a pulse is $0.29 \pm 0.02$. The bars $(\mathrm{b}$ in Fig. 2) show the calculated photon number distribution of weak coherent light (WCL) with the same average photon number. The sub-Poisson characteristics of our single-photon source are clearly observed, since the $P(1)$ of G-SPDC is higher than that of WCL and at the same time $P(2)$ of G-SPDC is much smaller than that of WCL.

The probability $P(2)$ of two-photon states indicates the security risk for quantum cryptography. The bars (c) in Fig. 2 show the photon number distribution of WCL having a $P(2)$ of 0.011 , which is the same as that of our source. The $P(1)$ of our source is twice as large as that of the WCL. This result also illustrates the potential advantage of our source over WCL as a single-photon source for quantum cryptography. This is our present experimental status. Next, we will analyze this photon number distribution quantitatively.
The probability $P(1)$ is restricted by the optical loss in the path of signal photons in our experimental setup. The $41 \%$ overall transmittance of the path suggests that the collection efficiency of the parametric fluorescence by the fiber coupler is $68 \%$, which is consistent with the estimation by independently performed measurement of the absolute quantum efficiency of the detection setup. ${ }^{20}$

Ideally, we can also eliminate the probability $P(2)$ to find more than two photons in a pulse by use of the G-SPDC method. $P(2)$ is not ideal, because the optical shutter used in our experiment is not perfect in two points. One is the noninfinitesimal shutter opening time (50 ns), and the other is the small leakage of photons through the closed optical shutter $(0.1 \%)$.

The following is a discussion of the possibility for applications that require higher repetition rates. One of the applications could be quantum key distribution (QKD). Note that long shutter opening time $(100 \mu \mathrm{s})$ in the present setup would be one of the problems that prevent an immediate adoption of our source for conventional QKD systems at $1.55-\mu \mathrm{m}$ wavelength with photon counters gated by nanosecond timing pulses. In our experiment, the repetition rate of $10 \mathrm{kHz}$ was limited by a photon pair emission rate of $10^{6}$ counts per second with a pump power of $200 \mathrm{~mW}$. With this photon pair emission rate, we had eight detection signals of control photons per pulse, on average. We can increase the repetition rate to several tens of kilohertz with our present setup; however, the likelihood that no control photon in a pulse can be found becomes significant when the repetition rate is increased too much. To overcome this limitation, one may be able to increase the photon emission rate up to $10^{9}$ counts per second by use of a periodically poled lithium niobate crystal $^{21}$ and an increased pump power of $1 \mathrm{~W}$. This means that the repetition rate of several tens of megahertz is possible with conventional technologies. This rate can compete with most single-photon sources using single-light emitters. Note also that none of the single-photon sources that have been reported so far can be used to replace the WCL in the conventional $1.55-\mu \mathrm{m}$ QKD system, because of available wavelength, low collection efficiencies, limited lifetimes, low operation temperatures, and so on.

Our G-SPDC method has two significant advantages over other kinds of single-photon sources. First, our method operates at room temperature and is very stable. This compares favorably with the fact that many other proposals suffer from a short lifetime or require low temperature operation. The other advantage is that the wavelength of single photons can be continuously changed. The SPDC process occurs for not only the degenerate condition but also other pairs of wavelengths when energy and momentum conservation laws are satisfied. In our case, we can easily change the wavelength of photons just by changing the angle of the $\beta$-barium borate crystal. This tunability is a unique characteristic of the G-SPDC method, since methods using single-light emitters have emission 
wavelengths that are determined by the materials or by the device structure.

Because we adopted a multimode fiber optical delay line to minimize the optical losses, the single photons are emitted in multimodes in space. We could not measure the bandwidth of the emitted photons with our present experimental setup, but we suppose that it should be $\sim 0.26 \mathrm{~nm}$ (FWHM). This is because we used a narrowband filter of $0.26 \mathrm{~nm}$ for control photons, and the corresponding signal photons that are emitted through the shutter should have a similar bandwidth owing to energy conservation. In this case, the associated coherence time of the emitted photons would be $6.25 \mathrm{ps}$. Therefore the photonic wave function is supposed to be localized in time in the whole time duration $(100 \mu \mathrm{s})$ of the pulses.

Note that even a source that emits single photons in multimode may be used in quantum cryptography experiments, such as the one recently reported using a built-in multimode optical fiber telecommunication network. ${ }^{22}$ The generation of single photons in a single mode in space and/or in a single mode in a time domain remains a challenge. ${ }^{23,24}$

\section{Conclusions}

In conclusion, we have experimentally demonstrated the generation of single-photon pulses using the G-SPDC method. The measured single-photon probability was found to be $P(1)=0.265$ at the collimated output port at a repetition rate of $10 \mathrm{kHz}$ after the effective quantum efficiency of $27.4 \%$ in the detection setup was compensated. The photon number distribution of the output pulses also clearly showed subPoissonian characteristics. Single-photon sources using our method have long lifetimes at room temperature and can be tuned to a wide range of frequencies.

We thank K. Ushizaka for assistance with the gate control circuit; K. Tsujino for assistance; Y. Yamamoto and E. Waks for their motivation; and H. F. Hofmann, H. Ohashi, H. Fujiwara, and J. Hotta for discussion. This work was supported by Japan Science and Technology Agency, Precursory Research for Embryonic Science and Technology project, Mitsubishi Electric, International Communications Foundation and Grant-in-Aid for Scientific Research(B) 12555008 of Japan Society for the Promotion of Science.

\section{References and Notes}

1. C. H. Bennett and G. Brassard, "Quantum cryptography: public key distribution and coin tossing," in Proceedings of IEEE International Conference on Computers, Systems and Signal Processing (IEEE Press, Los Alamitos, California, 1984), pp. 175-179.

2. E. Waks, A. Zeevi, and Y. Yamamoto, "Security of quantum key distribution with entangled photons against individual attacks," Phys. Rev. A 65, 052310 1-16 (2002).

3. F. De Martini, G. Di Giuseppe, and M. Marrocco, "Single-mode generation of quantum photon states by excited single molecules in a microcavity trap," Phys. Rev. Lett. 76, 900-903 (1996).

4. F. Treussart, R. Alleaume, V. Le Floc'h, L. T. Xiao, J.-M. Courty, and J.-F. Roch, "Direct measurement of the photon statistics of a triggered single photon source," Phys. Rev. Lett. 89, 093601 (2002).
5. C. Kurtsiefer, S. Mayer, P. Zarda, and H. Weinfurter, "Stable solid-state source of single photons," Phys. Rev. Lett. 85, 290 293 (2000).

6. C. Santori, M. Pelton, G. Solomon, Y. Dale, and Y. Yamamoto, "Triggered single photons from a quantum dot," Phys. Rev. Lett. 86, 1502-1505 (2001).

7. J. Kim, O. Benson, H. Kan, and Y. Yamamoto, "A single-photon turnstile device," Nature 397, 500-503 (1999).

8. Z. Yuan, B. E. Kardynal, R. M. Stevenson, A. J. Shields, C. J. Lobo, K. Cooper, N. S. Beattie, D. A. Ritchie, and M. Pepper, "Electrically driven single photon source," Science 295, 102105 (2002)

9. M. Pelton, C. Santori, J. Vuckovic, B. Zhang, G. S. Solomon, J. Plant, and Y. Yamamoto, "Efficient source of single photons: a single quantum dot in a micropost microcavity," Phys. Rev. Lett. 89, 233602 1-4 (2002).

10. C. K. Hong and L. Mandel, "Experimental realization of a localized one-photon state," Phys. Rev. Lett. 56, 58-60 (1986).

11. H. P. Yuen, "Generation, detection, and application of highintensity photon-number-eigenstate fields," Phys. Rev. Lett. 56, 2176-2179 (1986).

12. P. R. Tasper, J. G. Rarity, and J. S. Satchell, "Use of parametric down-conversion to generate sub-Poissonian light," Phys. Rev. A 37, 2963-2967 (1986).

13. J. G. Rarity, P. R. Tasper, and E. Jakeman, "Observation of sub-Poissonian light in parametric down conversion," Opt. Commun. 62, 201-206 (1987).

14. J. Mertz, A. Heidmann, C. Fabre, E. Giacobino, and S. Reynaud, "Observation of high-intensity sub-Poissonian light using an optical parametric oscillator," Phys. Rev. Lett. 64, 2897-2900 (1990).

15. For example, a single-photon source with the photon number distribution $\{P(0), P(1), P(2)\}=\{0.7,0.3,0\}$ is much more useful for quantum cryptography than that with $\{0.5,0.4$, $0.05\}$, where $P(n)$ is the probability to find $n$ photons in a pulse. Those photon number distributions, however, yield the same Fano factor $F=0.7$.

16. L. Mandel and E. Wolf, Optical Coherence and Quantum Optics (Cambridge U. Press, Cambridge, 1995), pp. 1074-1093.

17. S. Takeuchi, "Beamlike twin-photon generation by use of type 2 parametric down conversion," Opt. Lett. 26, 843-845 (2001).

18. C. H. Monken, P. H. S. Ribeiro, and S. Padua, "Optimizing the photon pair collection efficiency: a step toward a loopholefree Bell's inequalities experiment," Phys. Rev. A 57, R2267R2269 (1998).

19. This assumption was verified by measuring the coincidence by use of Hanbury-Brown and Twiss-type setups.

20. A. L. Migdall, R. U. Datla, A. V. Sergienko, J. S. Orszak, and Y. H. Shih, "Absolute detector quantum-efficiency measurements using correlated photons," Metrologia 32, 479-483 (1995/96).

21. K. Sanaka, K. Kawahara, and T. Kuga, "New high-efficiency source of photon pairs for engineering quantum entanglement," Phys. Rev. Lett. 86, 5620-5623 (2001).

22. T. Yoshikawa, N. Namekata, and S. Inoue, "Quantum key distribution using a telecommunication (multi-mode) fiber network," in Proceedings of the 7th Conference on Quantum Information Technology (Quantum Information Technology of the Institute of Electronics, Information and Communication Engineers, Tokyo, Japan, 2002), pp. 161-164.

23. S. Takeuchi, "Twin photon beams for the single photon generation," in Proceedings of the 7th International Symposium on Foundations of Quantum Mechanics in the Light of New Technology, ISQM-Tokyo '01 (World Scientific, Teaneck, N.J., 2002), pp. 98-103.

24. T. B. Pittman, B. C. Jacobs, and J. D. Franson, "Single photons on pseudo demand from stored parametric down-conversion," Phys. Rev. A 66, 042303 1-7 (2002). 